\title{
Artículos
}

\section{El uso de buenas prácticas agrícolas ¿Un problema de economías de escala? Estudio de caso de los productores de la Subcuenca III, Nicaragua}

\section{Alder Miguel Contreras Hernández*}

\section{Recibido: febrero 2015 / Aceptado: mayo de 2015}

Los riesgos del cambio climático y las debilidades productivas que presentan los pequeñosy medianos productores suponen un enorme reto en la agenda de crecimiento y desarrollo del sector agrícola nicaragüense. En este sentido, la incorporación de buenas prácticas agrícolas (GAP) a los sistemas de producción vigentes supone una posible estrategia de desarrollo para los grupos de productores más vulnerables, pues permite el acceso a mercados agrícolas más dinámicos y estables y la mejora de los sistemas productivos vigentes. No obstante, la implementación de sistemas de producción basados en principios de sostenibilidad económica y ambiental, como son los basados en las GAP, supone enormes desafíos tanto económicos como culturales, por lo que el desarrollo de políticas de intervención que permitan su establecimiento y aplicación es de vital importancia para el desarrollo del sector agrícola nicaragüense.

Palabras clave: buenas prácticas agrícolas / sector agrícola / desarrollo productivo

\section{Introducción}

La implementación de buenas prácticas agrícolas (GAP, por sus siglas en inglés) ha venido adquiriendo gran importancia en la mayoría de los países agrícolas, dada la creciente demanda de productos inocuos, orgánicos, con altos estándares 
de calidad y cuyo método de producción esté cimentado en principios de sostenibilidad económica y ambiental (Meer, 2006; Poisot, Speedy, \& Kueneman, 2004; Pongvinyoo, Yamaho, \& Hosono, 2014). Sin embargo, son muchos los factores que limitan la implementación de las GAP, sobre todo en aquellos países poco desarrollados donde las capacidades económico-productivas de los agricultores y los altos estándares internacionales de calidad provocan procesos de exclusión que restringen la inserción de los grupos de productores vulnerables a mercados más dinámicos y a los procesos inherentes de transferencia y difusión tecnológica (Pongvinyoo, Yamaho, \& Hosono, 2014; Vieira, 2013; Yoguel, 2000).

En la presente investigación se construye un índice de capacidad de los productores agrícolas de la Subcuenca III para implementar buenas prácticas agrícolas (ICGAP), con el fin de analizar su distribución y los factores que lo determinan. Para estimar el ICGAP se utiliza el modelo logístico de un parámetro (1PL, por sus siglas en inglés), perteneciente a la familia de los modelos derivados de la teoría de respuesta (ITR, por sus siglas en inglés). Los modelos de la denominada ITR se utilizan normalmente en el campo de la educación para crear índices de valoración cuantificables de variables con alto componente subjetivo, tales como la inteligencia, a partir de variables de naturaleza binomial y/o categórica (Zheng \& Rabe-Hesketh, 2007). Así entonces, en el contexto de la presente investigación se utilizan variables de naturaleza binomial que indican la utilización o no utilización de determinada práctica agrícola para medir la capacidad de implementarlas.

El objetivo principal del presente documento es determinar si la capacidad económico-productiva de los productores agrícolas del territorio analizado constituye un factor significativo en su nivel de capacidad para implementar las GAP. Para ello, el documento se divide en cinco partes, incluyendo la presente introducción. En la segunda parte se discute brevemente el concepto de GAP y su importancia. En la tercera parte se presenta la metodología utilizada, para luego, en la cuarta parte, analizar los principales resultados. Por último, en la quinta parte se exponen las principales conclusiones y recomendaciones derivadas del estudio.

\section{El concepto de buenas prácticas agrícolas}

El concepto de GAP, introducido por la Organización de las Naciones Unidas para la Alimentación y la Agricultura (FAO, por sus siglas en inglés), consiste en un sistema de aplicación voluntaria orientado a pequeños productores y basado en prácticas de producción agrícola sostenibles y amigables con el medio ambiente (Meer, 2006; Poisot, Speedy, \& Kueneman, 2004). Actualmente, el concepto de GAP se utiliza en gran número de países agrícolas con el objetivo de incrementar la competitividad, la seguridad alimentaria y la estandarización de los productos agrícolas. Sin embargo, es común que la mayoría de productores no puedan aplicarlo, debido al poco desarrollo económico-productivo que poseen y al rigor de las normas de comercio internacional, además de la presencia de fuertes barreras culturales que impiden sustituir los métodos y sistemas de producción vigentes (Pongvinyoo, Yamaho, \& Hosono, 2014).

Si bien es cierto que existe un consenso sobre la importancia de implementar GAP en los procesos de producción agrícola, aún se debate cuáles prácticas agrícolas 
deben considerarse buenas. Al respecto, Pongvinyoo, Yamaho y Hosono (2014) sostienen que su definición es contextual, es decir, la consideración de una práctica agrícola como GAP depende del entorno socio-económico y del tipo de actividad agrícola en que se desenvuelven los productores. Dada esta subjetividad en cuanto a la definición, en este documento se considerarán como GAP las establecidas por el Ministerio de Agricultura y Forestal (MAGFOR), mismas que se resumen en el Cuadro 2.1.

Cuadro 2.1: Prácticas agrícolas analizadas

\begin{tabular}{cc}
\hline \multicolumn{2}{c}{ Práctica agrícola } \\
\hline Curvas de nivel & Prácticas postcosecha \\
Control de plagas y enfermedades & No quema \\
Barreras de retención & Elaboración de abono orgánico \\
Cultivos de cobertura & Rotación de cultivos \\
Cercas vivas & Ronda contra incendios \\
Cero labranza & Limpia y poda \\
Barreras rompeviento & \\
\hline
\end{tabular}

Fuente: Elaboración propia.

Así entonces, en la siguiente parte del estudio se construirá una medida de la capacidad de implementación de las GAP (ver Cuadro 2.1) en el caso específico de los productores agrícolas de la Subcuenca III del municipio de Managua, para luego analizar su distribución y sus principales determinantes.

\section{Metodología}

\subsection{Los datos}

El análisis comprende un total de 3,852 explotaciones agropecuarias (EA) dedicadas principalmente a actividades agrícolas ubicadas en la Subcuenca III, situada entre los departamentos de Managua y Masaya (ver Figura 3.1). La información individual de las EA proviene del IV Censo Nacional Agropecuario (CENAGRO), el cual se encuentra disponible en la página web oficial del Instituto Nacional de Información y Desarrollo (INIDE; www.inide.gob.ni). 
Figura 3.1: Ubicación geográfica de la Subcuenca III.

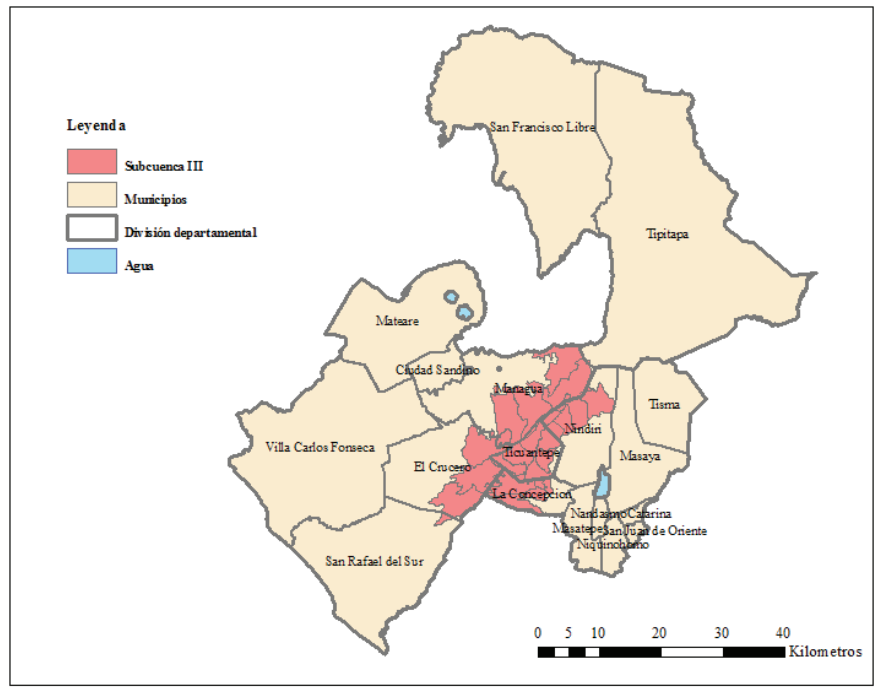

Fuente: Elaboración propia.

\subsection{El modelo de Rasch: modelo logístico de un parámetro}

Para analizar la distribución y los factores que determinan la capacidad latente de cada productor para implementar distintas prácticas agrícolas es necesario crear un índice o indicador que permita cuantificar dicha capacidad. Sin embargo, es evidente que la variable que se trata de medir -capacidad- es una variable no observable que posee un carácter altamente subjetivo. Para solucionar este problema y obtener una medida cuantificable de la capacidad de implementar prácticas agrícolas se utiliza como marco de referencia la teoría de respuestas (ITR, por sus siglas en inglés), empleando variables de naturaleza dicótoma que adquieren el valor de 1 cuando se utiliza determinada práctica agrícola, y de 0 cuando no se utiliza (ver Cuadro 2.1). Estos modelos se basan en la idea de que el rasgo latente del individuo analizado está relacionado con una serie de indicadores, que en nuestro caso vienen representados por las variables dicótomas. Así, en el caso de los productores analizados, el uso o desuso de determinada práctica agrícola puede servir como parámetro para medir su capacidad para implementar determinada práctica agrícola y su respectiva dificultad de implementación.

Uno de los modelos de la ITR más conocido es el denominado modelo de Rasch, propuesto en un principio por Rasch (1960) y posteriormente desarrollado por Wright (1977) y Fischer (1995). En este modelo, la probabilidad de que sea correcta la respuesta al elemento $i$-utilización o no de alguna práctica agrícola- dada por el individuo $n$-productor- es modelada como una función de un parámetro $\delta i$, asociado al elemento, el cual representa su respectiva dificultad; y un parámetro $\theta n$, asociado al individuo, el cual representa su rasgo latente (ver Ecuación 3.1).

$$
\operatorname{Pr}\left(x_{i n}=1 \mid \theta_{n}\right)=\frac{e^{\left(\delta_{i}-\theta_{n}\right)}}{1+e^{\left(\delta_{i}-\theta_{n}\right)}}
$$


El modelo de la ecuación 3.1 también se conoce como el modelo logístico de un parámetro $(1 \mathrm{PL})$, porque solo existe un parámetro $\delta i$ por elemento. En dicho modelo, la probabilidad de que la respuesta a un elemento sea correcta aumenta con la habilidad del individuo, disminuye con la dificultad del elemento y es igual a 0.5 cuando la habilidad del individuo y la dificultad del elemento son iguales. Así entonces, en el contexto de la presente investigación el parámetro $\delta i$ representa la dificultad individual de implementar $i$-ésima práctica agrícola (ver Cuadro 2.1) y el parámetro $\theta n$ representa la capacidad del $n$-ésimo productor para implementar dichas GAP.

\section{Resultados}

\section{1 Índice de capacidad para implementar prácticas agrícolas}

Las estimaciones del modelo 1PL se realizaron a partir del método propuesto por Zheng y Rabe-Hesketh (2007), cuyos resultados se muestran en el Cuadro 4.1. Los coeficientes de cada práctica agrícola representan, respectivamente, su dificulad de implementación, donde los valores que se localizan a la derecha del cero absoluto representan mayor dificultad y los valores que se localizan a la izquierda del cero abosoluto representan la menor dificultad. De este modo, se puede inferir que la implementación de cero labranza, cultivos alternativos, prácticas postcosecha y elaboración de abono orgánico son las prácticas más dificiles de implementar para los producteres analizados. Por otro lado, la utilización de cercas vivas, control de plagas y enfermedades y rondas contra incendios son las prácticas más comunes y más fáciles de implementar.

Cuadro 3.1: Estimación del modelo 1PL

\begin{tabular}{lccc}
\hline Práctica agrícola & Coeficiente & Str. Err. & $P>|z|$ \\
\hline Cero labranza & 4.55 & 0.12 & 0.00 \\
Cultivos de cobertura & 4.44 & 0.12 & 0.00 \\
Prácticas postcosecha & 3.82 & 0.92 & 0.00 \\
Elaboración de abono orgánico & 3.52 & 0.08 & 0.00 \\
Rotación de cultivos & 2.98 & 0.07 & 0.00 \\
Curvas de nivel & 2.47 & 0.06 & 0.00 \\
Barreras rompeviento & 2.47 & 0.06 & 0.00 \\
Barreras de retención & 2.44 & 0.06 & 0.00 \\
Limpia y poda & 2.20 & 0.05 & 0.00 \\
No quema & 1.90 & 0.05 & 0.00 \\
Cercas vivas & 1.37 & 0.05 & 0.00 \\
Control de plagas y enfermedades & 0.91 & 0.04 & 0.00 \\
Ronda contra incendios & -2.05 & 0.05 & 0.00 \\
\hline
\end{tabular}

Fuente: Elaboración propia. 
Dadas las estimaciones del modelo 1PL, el ICGAP asociado a cada productor se calcula según el método propuesto por Zheng y Rabe-Hesketh (2007) (ver Ecuación 3.2).

$$
E\left(\theta_{n} \mid \boldsymbol{Y}_{n}\right)=\int_{-\infty}^{\infty} \theta_{n}\left\{\prod_{i=1}^{I} \operatorname{Pr}\left(\boldsymbol{y}_{\text {in }} \mid \theta_{n}\right)\right\} g\left(\theta_{n}\right) d \theta_{n}
$$

Los resultados de las estimaciones del ICGAPs se muestran en el histograma de la Figura 4.1, el cual revela la existencia de un claro sesgo hacia la derecha, indicando que la gran mayoría de productores poseen poca capacidad para implementar GAP. Esto evidencia la necesidad de establecer estímulos que permitan una mayor difusión e implementación de GAP, con el objetivo de mejorar y hacer más rentable el proceso de producción y, a la vez, lograr que estos estímulos sean sostenibles a nivel económico y medioambiental.

Figura 4.1: Distribución del ICGAP entre productores.

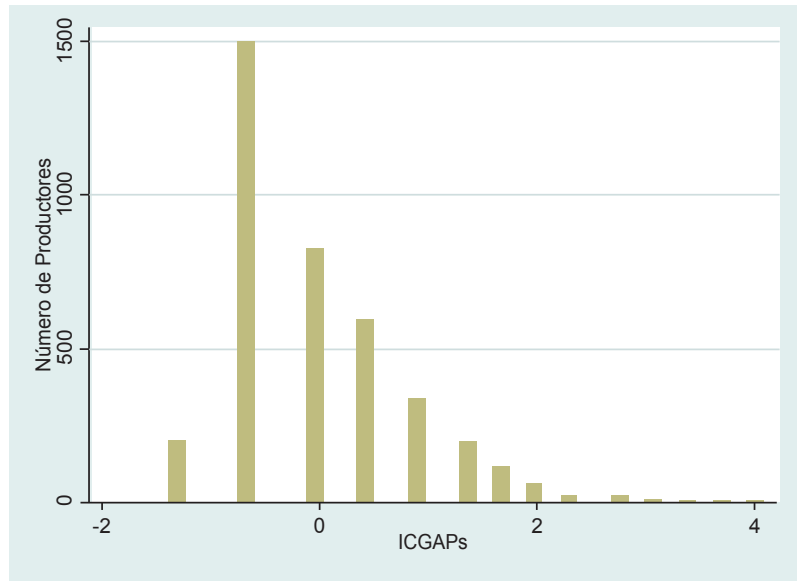

Fuente: Elaboración propia.

La desigual distribución de las capacidades para implementar GAP observada en los productores de la Subcuenca III podría deberse, como señalan Pongvinyoo, Yamaho y Hosono (2014) y Vieira (2013), a su escaso desarrollo económicoproductivo y a la poca capacidad que poseen para adquirir, implementar y transmitir nuevas capacidades productivas. Así entonces, en la siguiente parte se analiza el efecto del nivel de desarrollo económico-productivo de cada agricultor en su nivel de capacidad para implementar GAP, a fin de determinar si el problema concerniente a su aplicación obedece a factores relacionados con los procesos de economías de escala propios de los métodos de producción agrícola, o si por el contrario, puede ser implementado indistintamente del nivel de desarrollo de los productores. 


\subsection{La tenencia de capital tierra y la capacidad de implementar prácticas agrícolas}

Como se dijo anteriormente, el objetivo principal de esta investigación es determinar si el nivel de desarrollo económico-productivo inherente a cada productor influye de manera significativa en su capacidad para implementar GAP. Sin embargo, el CENAGRO no brinda información sobre los niveles de productividad individual de los productores y, por ende, tampoco sobre el volumen de ingresos que estos obtienen de sus actividades agrícolas. Dada esta limitante, en este documento se utiliza como variable proxy del volumen de ingreso y de capacidad económicoproductiva la cantidad de capital tierra que los agricultores poseen, puesto que, en la mayoría de los casos, la cantidad de tierra que un productor agrícola posee representa una proporción significativa de su riqueza (Ray, 2003).

Así entonces, es de esperar que cuanto mayor sea la cantidad de tierra de que disponen los productores, mayor será su capacidad de implementar GAP. Para verificar esta hipótesis se procede primero a crear agrupaciones de productores según la cantidad de tierra, utilizando para ello el método de clusterización jerárquica propuesto por Ward (1963). Este método de agrupación permitirá obtener conjuntos de productores con niveles de tenencia de capital tierra $-\mathrm{y}$, por ende, niveles de desarrollo económico-productivo- más o menos similares a lo interno de un mismo conjunto, pero distintos entre productores de diferentes conjuntos (Rencher \& Christensen, 2012). Los resultados del proceso de formación de clusters se muestran en el dendrograma de la Figura 4.2, elaborada sobre un total de 20 ramificaciones, G .

Figura 4.2: Dendrograma del proceso de clusterización de productores según su tenencia de tierra.

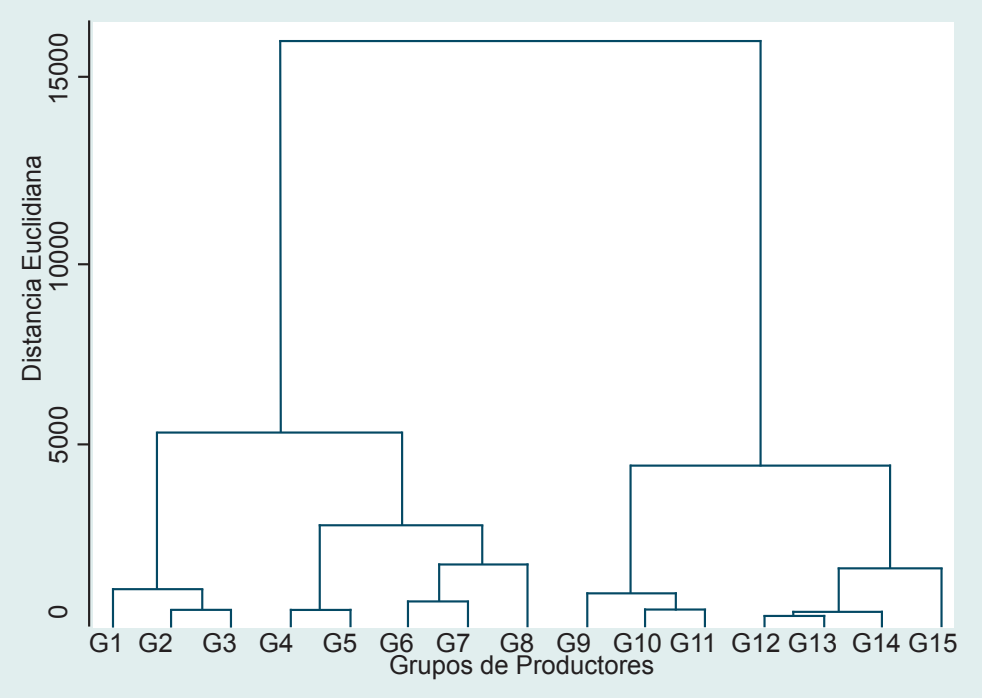

Fuente: Elaboración propia. 
Según el análisis de cluster anterior, existen cuatro agrupaciones de productores relevantes (ver Figura 4.2) a las cuales denominaremos $A, B, C$ y D, respectivamente. Estas agrupaciones poseen diferencias significativas en cuanto a la tenencia de capital tierra, indicando una fuerte desigualdad en la distribución de la misma. Como se muestra en el Cuadro 4.2, la mayoría de los productores se concentran en las agrupaciones $A$ y $B$, las cuales poseen, en promedio ${ }^{1}$, la menor cantidad de tierra, $0.9 \mathrm{mz}$ y $8.2 \mathrm{mz}$, respectivamente. Por su parte, existe un número pequeño de productores que poseen grandes extensiones de tierra, aglomerados en los grupos C y D, con un promedio $113.3 \mathrm{mz}$ y $350.2 \mathrm{mz}$, respectivamente. Por lo tanto, es evidente que en el territorio analizado existe un alto nivel de desigualdad en cuanto a la distribución de la tierra, el cual podría incidir directamente en su capacidad para implementar GAP.

Cuadro 4.2: Tenencia de la tierra entre grupos de productores

\begin{tabular}{|c|c|c|c|c|}
\hline Grupos & Cantidad de productores & Promedio manzanas & Str. Err. & $P>|z|$ \\
\hline & 2664 & 0.92 & 0.25 & 0.00 \\
\hline & 1113 & 8.25 & 0.38 & 0.00 \\
\hline & 55 & 113.26 & 1.73 & 0.00 \\
\hline & 20 & 350.24 & 2.87 & 0.00 \\
\hline
\end{tabular}

Fuente: Elaboración propia.

Dada la desigual distribución de la tierra observada en el territorio (ver Cuadro 4.2), es de esperar que existan también grandes disparidades en cuanto a los niveles de desarrollo económico-productivo, lo que podría explicar gran parte de la desigualdad de los productores en cuanto a la capacidad de implementar prácticas agrícolas (ver Figura 4.1). De hecho, como se muestra en el Cuadro 4.3, el valor promedio del ICGAP2 aumenta conforme nos desplazamos del grupo A al C, lo que evidencia una clara relación positiva entre el grado de desarrollo económicoproductivo con que el que cuenta la EA y la capacidad de los productores para implementar GAP. Sin embargo, es notorio que los productores del grupo $D$, aquellos con mayores extensiones de tierra, no poseen, en promedio, los valores más altos del índice. Por otro lado, llama la atención también que en todos los grupos existen productores que poseen los valores mínimos del índice de capacidad, mientras que los valores más altos parecen presentarse tanto en productores pequeños como en grandes productores. Por estas razones se puede inferir que el grado de desarrollo económico-productivo afecta el nivel de capacidad de implementar GAP, pero no constituye un factor indispensable o restrictivo.

1 El cálculo del promedio de manzanas según el grupo de productores se realizó utilizando un modelo de análisis de varianza (ANOVA), el cual, además de brindar el nivel promedio de manzanas, otorga un estadístico de prueba para determinar si dichas medias son estadísticamente diferentes. En este caso, todas las medias fueron estadísticamente significativas, por lo que la diferencia en la tenencia de tierra entre grupos es relevante.

2 Al igual que con el cálculo del promedio de manzanas por tipo de productor, el promedio del índice de capacidad de implementar prácticas agrícolas se realizó utilizando un modelo ANOVA. 
Cuadro 4.3: Índice de capacidad de implementar PA, por grupos de productores

\begin{tabular}{cccccc}
\hline Grupos & ICGAP promedio & Mínimo & Máximo & Str. Err. & $P>|z|$ \\
\hline-0.17 & -1.37 & 3.72 & 0.01 & 0.00 \\
0.36 & -1.37 & 4.10 & 0.02 & 0.00 \\
0.89 & -1.37 & 3.72 & 0.11 & 0.00 \\
0.75 & -1.37 & 2.70 & 0.18 & 0.00 \\
\hline
\end{tabular}

Fuente: Elaboración propia.

De este modo, la implementación de un modelo de producción agrícola basado en GAP parece ser una opción posible y viable para mejorar la condición socioeconómica de aquellos grupos de productores más vulnerables que poseen pocos recursos. En este sentido, la implementación de prácticas agrícolas amigables con el medio ambiente y sostenibles desde el punto de vista económico que permitan la producción de productos orgánicos de alta calidad constituye una gran oportunidad de acceso para los pequeños y medianos productores a mercados más selectos que ofrecen mejores precios. Sin embargo, son estos grupos de productores los que presentan menores valores del ICGAP en términos de frecuencia (ver Figura 4.2), observándose en su distribución un claro sesgo hacia la derecha que luego desaparece paulatinamente conforme nos desplazamos hacia los grupos con mayor tenencia de recursos (ver Figura 4.3).

Figura 4.3: Distribución del ICGAP, por grupos de productores.

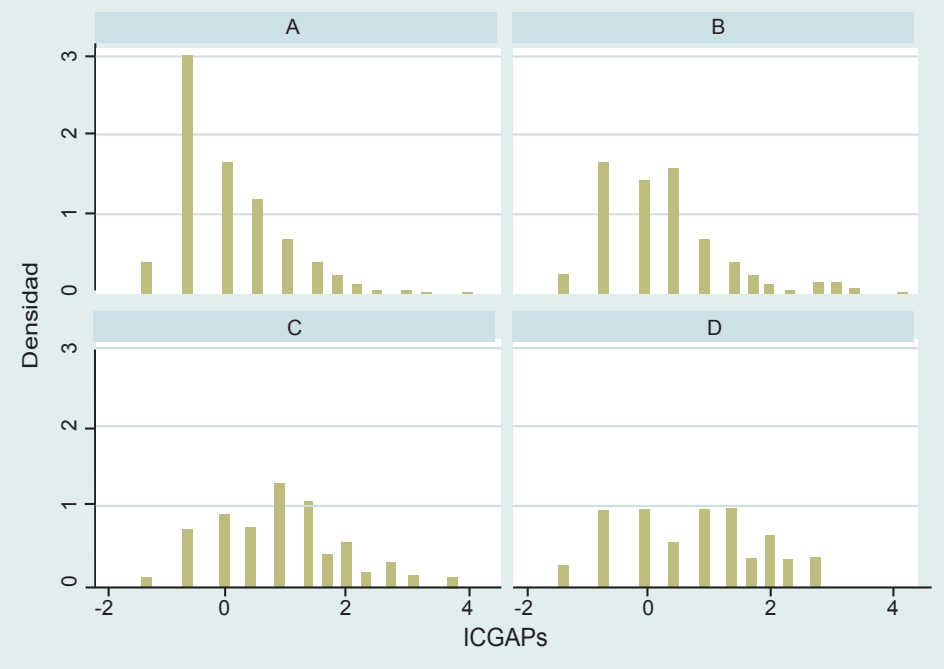

Fuente: Elaboración propia. 
La presencia de bajos niveles del ICGAP en los pequeños productores supone un gran desafío en términos de políticas que incentiven la aplicación de GAP, sobre todo por el hecho de que la mayoría de productores suelen ser reacios a realizar cambios bruscos en sus sistemas productivos, dado el tiempo que llevan implementándolos y la experticia derivada de procesos de aprendizaje empíricos. En este sentido se puede afirmar, según los resultados de la presente investigación, que la capacidad de un productor para implementar prácticas agrícolas (PA) depende en cierto modo de su grado de desarrollo económico-productivo; sin embargo, esta característica no es exclusiva de aquellos productores grandes, sino que es un modelo productivo que también pueden aplicar los pequeños y medianos productores.

\section{Conclusiones y recomendaciones}

La gran mayoría de los productores agrícolas pertenecientes a la Subcuenca III presentan muy poca capacidad para implementar GAP, lo que se explica en cierta medida por la poca capacidad económico-productiva que poseen. Al respecto, los resultados muestran un claro sesgo en la forma en que se distribuye el ICGAP estimado, donde la gran mayoría de los productores analizados, principalmente los pequeños y medianos, presentan muy bajos valores del índice, siendo estos, además, los que tienen menos disponibilidad de capital tierra. No obstante, se puede apreciar también que los valores más altos del ICGAP están distribuidos indistintamente de la clasificación de los grupos según su capacidad económico-productiva, lo que muestra que este factor no constituye un factor restrictivo para implementar GAP, lo que hace de este un modelo productivo que puede ser adoptado por los productores pequeños y medianos.

Así entonces, es evidente la necesidad de establecer mecanismos de intervención, principalmente de naturaleza pública, que permitan crear incentivos para la incorporación de GAP en el actual modelo productivo, sobre todo para aquellos productores agrícolas pequeños y medianos del territorio analizado. Estos incentivos deben estar orientados a fortalecer y crear capacidades productivas que incorporen GAP, así como también estrategias organizativas que faciliten su inserción a mercados más dinámicos que les permitan obtener réditos bastantes para asentar los nuevos métodos de producción. Por último, es recomendable que el desarrollo de tales políticas de intervención esté cimentado en estudios más específicos que permitan analizar de manera más concreta el entorno local de los productores del área y sus vínculos con los demás actores involucrados en las cadenas productivas y de valor a las que se insertan.

\section{Referencias bibliográficas}

Fischer, G. (1995). Rasch Models: Foundation, Recent Developments and Application. New York: Springer.

Meer, V. (2006). Exclusion of Small-Scale Farmers from Coordinated Supply Chain: Market Failure, Policy Failure or Just Economies of Scale? In R. Ruben, M. Slingerland, \& H. Nijhoff, Agro-food Chains and Networks for Development (pp. 
209-217). Netherlands: Springer.

Poisot, A. S., Speedy, A., \& Kueneman, E. (2004). Incentive for the Adoption of Good Agricultural Practices, Background Paper for the FAO Expert Consultation on a Good Agricultural Practices Approach. Rome.

Pongvinyoo, P., Yamaho, M., \& Hosono, K. (2014). Factors affecting the implementation of good agricultural practices (GAP) among coffee farmers in Chumphon Province, Thailand. American Journal of Agricultural Development, 2, 34-39.

Rasch, G. (1960). Probabilistic Models for Some Intelligence and Attainment Tests. Copenhagen: Danmarks Pædagogiske Institut.

Ray, D. (2003). Economía del Desarrollo. Barcelona: Antoni Bosch Editor.

Rencher, A., \& Christensen, W. (2012). Methods of Multivariate Analysis (3nd ed.). NJ: Wiley.

Vieira, J. E. (2013). Heterogeneidad estructural de la agricultura familiar en Brasil. Revista CEPAL, 111, 103-121.

Ward, J. (1963). Hierarchical grouping to optimize an objective function. Journal of the American Statistical Association, 58, 236-244.

Wright, B. (1977). Solving measurement problems with the Rasch model. Journal of Educational Measurements, 14, 97-116.

Yoguel, G. (2000). Creación de competencias en ambientes locales y redes productivas. Revista CEPAL, 77, 105-119.

Zheng, X., \& Rabe-Hesketh, S. (2007). Estimating parameters of dichotomous and ordinal response models with gllamm. The Stata Journal, 3, 313-333. 